\title{
Universal and non-universal renormalizations in Fermi liquids
}

\author{
Andrey V. Chubukov ${ }^{1}$ and Dmitrii L. Maslov ${ }^{2}$ \\ ${ }^{1}$ Department of Physics, University of Wisconsin-Madison, 1150 University Ave., Madison, WI 53706-1390 \\ ${ }^{2}$ Department of Physics, University of Florida, P. O. Box 118440, Gainesville, FL 32611-8440
}

(Dated: August 14, 2018)

\begin{abstract}
We discuss an interplay between the Fermi-liquid (FL) theory and diagrammatic perturbative approach to interacting Fermi systems. In the FL theory for Galilean-invariant systems, mass renormalization $\mathrm{m}^{*} / \mathrm{m}$ comes exclusively from fermions at the Fermi surface. We show that in a diagrammatic perturbation theory the same result for $m^{*} / m$ comes from fermions both at and away from the Fermi surface. The equivalence of the FL and pertubative approaches is based on a particular relation between self-energy contributions from high- and low-energy fermions. We argue that care has to be exercised in the renormalization group approach to a FL in order not to miss the high-energy contribution to $m^{*} / m$. As particular examples, we discuss $m^{*} / m$ and the quasiparticle residue $Z$ for $2 \mathrm{D}$ and $3 \mathrm{D}$ systems with both $S U(2)$ and $S U(N)$ symmetries, and with a short-range interaction. We derive an expression for the anisotropic part of the Fermi-liquid vertex in the large- $N$ limit of the $S U(N)$ case.
\end{abstract}

PACS numbers: 71.10.Hf, 71.27.+a

\section{INTRODUCTION}

Despite its apparent simplicity, the Landau Fermi Liquid (FL) theory is one of the most non-trivial theories of interacting fermions $\underline{\underline{1}} \underline{\underline{\underline{4}}}$ It states that the linewidth of a state near the Fermi surface (FS) is smaller than its energy, so that the quasiparticle propagator $G(\omega, \mathbf{p})$ has a well-defined pole at $\omega=p_{F}\left(|\mathbf{p}|-p_{F}\right) / m^{*}+\mathcal{O}\left(|\mathbf{p}|-p_{F}\right)^{2}$, where $p_{F}$ is the Fermi momentum. It also states that the quasiparticle residue $Z$ and effective mass $m^{*}$ are expressed in terms of an interaction vertex $\Gamma_{\alpha \beta, \gamma \delta}^{\omega}(p, q)$, where $p \equiv(\omega, \mathbf{p})$ and $q \equiv\left(\omega^{\prime}, \mathbf{q}\right)$, with one of the two "four-momenta" on the FS, e.g., $p \equiv\left(0, \mathbf{p}_{F}\right)$, where $\mathbf{p}_{F} \equiv$ $(\mathbf{p} /|\mathbf{p}|) p_{F}$. [The vertex $\Gamma_{\alpha \beta, \gamma \delta}^{\omega}$ is obtained from a fully renormalized, antisymmetrized vertex $\Gamma_{\alpha \beta, \gamma \delta}\left(p, q ; p_{1}, q_{1}\right)$ in the limit of zero momentum- and vanishing energy transfer, i.e., for $\left|\mathbf{p}_{1}\right|=|\mathbf{p}|,\left|\mathbf{q}_{1}\right|=|\mathbf{q}|, \omega_{1} \rightarrow \omega$, and $\omega_{1}^{\prime} \rightarrow \omega^{\prime}$. ] Finally, the FL theory states that, for a Galilean invariant system $\left(\mathbf{p}^{2} / 2 m\right.$ dispersion for a free particle), which is the only case considered in this paper, the effective mass $m^{*}$ is expressed via $\Gamma_{\alpha \beta, \gamma \delta}^{\omega}(p, q)$ with both four-momenta on the FS. On the other hand, renormalization of the quasiparticle residue $Z$ comes from fermions with $p=p_{F}$ but $q$, in general, is away from the FS.

Explicitly, for a Galilean-invariant system,,$\frac{1,5}{\Perp}$

$$
G_{p}=\frac{Z}{\omega-p_{F}\left(|\mathbf{p}|-p_{F}\right) / m^{*}},
$$

where

$$
\begin{gathered}
\frac{1}{Z}=1-\frac{i}{2} \sum_{\alpha \beta} \int \Gamma_{\alpha \beta, \alpha \beta}^{\omega}\left(p_{F}, q\right)\left(G_{q}^{2}\right)_{\omega} \frac{d^{D+1} q}{(2 \pi)^{D+1}}, \\
\frac{1}{m^{*}}=\frac{1}{m}-A_{D} \sum_{\alpha \beta} \int \Gamma_{\alpha \beta, \alpha \beta}^{\omega}\left(p_{F}, q_{F}\right) \frac{\mathbf{p}_{F} \cdot \mathbf{q}_{F}}{p_{F}^{2}} d \Omega_{q},
\end{gathered}
$$

$\Omega_{q}$ is the solid angle, $A_{D}=Z^{2} k_{F}^{D-2} / 2(2 \pi)^{D}, G_{q}$ is the full fermionic propagator, and $\left(G_{q}^{2}\right)_{\omega}$ is the product of the two Green's functions with the same momenta and infinitesimally close frequencies. Note that integration in Eq. (1.2b) is only over $d \Omega_{q}$, which implies that mass renormalization comes solely from fermions on the FS. In the field-theoretical language, mass renormalization is then a low-energy, universal phenomenon, while a reduction of $Z$ from its bare value of one is a high-energy, non-universal phenomenon.

The effective mass $m^{*}$ and $Z$ factor can also be obtained by expanding the self-energy $\Sigma\left(\omega, \epsilon_{\mathbf{p}}\right)$ to first order in $\omega$ and $\epsilon_{\mathbf{p}}$ :

$$
\Sigma\left(\omega, \epsilon_{\mathbf{p}}\right)=\left(\omega-\epsilon_{\mathbf{p}}\right)\left(\frac{1}{Z}-1\right)-\epsilon_{\mathbf{p}}\left(\frac{m}{m^{*}}-1\right)+\mathcal{O}\left(\omega^{2}, \epsilon_{p}^{2}\right) .
$$

[We define $\Sigma$ by $G_{p}^{-1}=\omega-\epsilon_{\mathbf{p}}+\Sigma\left(\omega, \epsilon_{\mathbf{p}}\right)$ with $\epsilon_{\mathbf{p}}=\left(\mathbf{p}^{2}-\right.$ $\left.p_{F}^{2}\right) / 2 m$.] As, in practice, the self-energy is obtained via a diagrammatic perturbation theory, we will refer to this approach as to "perturbative". In the earlier days of the FL theory, perturbative calculations were used as a check of the general FL relations, e.g., it has been verified ${ }^{6.7}$ that the values of $m^{*} / m$ and $Z$ in Eq. (1.3) are the same as in Eqs. 1.2a and 1.2b. However, whether mass renormalization in Eq. (1.3) comes from low energies, as it does in Eq. 1.2b), has not been verified.

In this paper we demonstrate that, in a diagrammatic calculation, mass renormalization is not, in general, a low-energy phenomenon. A low-energy contribution to $m^{*}$ does, indeed, exists, but there is also another, highenergy contribution. Only the sum of the two contributions reproduces the Landau formula for the effective mass, Eq. (1.2a). There are situations (see below) when the high-energy contribution is relatively small but, in general, it is of the same order as the low-energy one.

The reason why low-energy mass renormalization is generally not the full result in a diagrammatic calculation, can be traced back to the fact that the building 
block of diagrammatics is a non-antisymmetrized interaction potential $U(\mathbf{k})$ rather than the antisymmetrized vertex function $\Gamma^{\omega}$. An expression for $\Gamma^{\omega}$ in terms of $U(|\mathbf{k}|)$ does contain a high-energy contribution and, when the self-energy is expressed in terms of $U(|\mathbf{k}|)$ rather than in terms of $\Gamma^{\omega}$, these high-energy terms do contribute to the effective mass. When one re-expresses $\Sigma$ in terms of $\Gamma^{\omega}$, the high-energy contributions to $m^{*}$ cancel out.

An issue where mass renormalization comes from is important for the interpretation of a Fermi liquid as a fixed point of the momentum-space renormalization group ( $\mathrm{RG}$ ) transformation $\stackrel{8}{=}$ In the $\mathrm{RG}$ approach, one progressively integrates out high-energy fermions ending up with a renormalized interaction among low-energy ones. According to Eq. (1.2b), this interaction is all one needs to evaluate the effective mass. Our finding that, in a diagrammatic calculation, $\mathrm{m}^{*} / \mathrm{m}$ may have contributions from both low- and high-energy fermions implies that care has to be exercised in applying the RG approach to a FL. Specifically, we argue that to recover the Landau formula for $m^{*} / m$, one also has to take into account that, in the process of RG flow, the "bare" mass for the lowenergy theory changes from the free-fermion mass $m$ to a different value $\left(m_{B}\right)$. The difference $m_{B} / m-1$ comes from high energies. Only the sum of regular renormalization from $m$ to $m_{B}$ and low-energy renormalization of $m_{B}$ yields the agreement with the FL theory.

We further demonstrate that that there exists a certain identity [cf. Eq. (4.1)], which relates the high- and low-energy terms. This identity involves combinations of fermionic Green's functions in particle-hole and particleparticle channels, and is exact to first order in $\omega$ and $\epsilon_{\mathrm{p}}$. Adding this identity to the diagrammatic self-energy $\Sigma\left(\omega, \epsilon_{\mathbf{p}}\right)$ does not change $\mathcal{O}(\omega)$ and $\mathcal{O}\left(\epsilon_{\mathbf{p}}\right)$ terms in $\Sigma$, i.e., it does not change $Z$ and $m^{*} / m$, but, at the same time, it transforms the high-energy contribution into the low-energy one, and makes the diagrammatic self-energy equivalent to the self-energy extracted from the FL theory.

An interesting example of comparison between the FL and perturbative approaches is the large- $N$ limit for an $S U(N)$-invariant 2D system with short-range interaction. The perturbative self-energy in this case is obtained simply by retaining the Random-Phase Approximation
(RPA) diagrams with maximal number of particle-hole bubbles at each order in the interaction. It is not enough, however, to retain only diagrams with a maximal number of bubbles in order to construct $\Gamma^{\omega}$ because these diagrams contribute only to an isotropic part of $\Gamma^{\omega}$ and, therefore, do not lead to mass renormalization. We show that the perturbation theory for an anisotropic part of $\Gamma^{\omega}$ can be resummed to infinite order in $U$ even for subleading in $1 / N$ terms, and the resulting expression for $m^{*}$ coincides with that obtained from the self-energy.

The structure of this paper is as follows. In Sec. [1] we discuss the FL theory and perturbation series for $\Gamma^{\omega}$. We briefly discuss the 3D case, and present the FL expressions for $m^{*} / m$ and $Z$ in 2D with a short-range interaction (to the best of our knowledge, the result for $Z$ has not been derived in the prior literature.) In Sec. III. we obtain the self-energy in the diagrammatic perturbation theory both in 3D and 2D and identify the lowand high-energy contributions to the effective mass. We show that $m^{*} / m$ ad $Z$ are indeed the same as in the FL theory, but at least part of mass renormalization comes from high energies. Moreover, we show that, in 2D, entire mass renormalization to second order in the interaction comes from high energies, if the calculation is performed by combining internal fermions into particle-hole pairs, while the high-energy part is twice larger and of opposite sign to the low-energy part, if internal fermions are combined into particle-particle pairs. In Sec.IV] we reconcile the two approaches by proving a particular relation between the convolutions of Green's functions. In Sec. V we discuss an extension of our results to the $S U(N)$ case and consider the large $N$ limit. Finally, in Sec. VI we present our conclusions.

\section{FERMI-LIQUID THEORY}

\section{A. Pitaevskii-Landau relations}

We remind the reader that Eqs. (1.2a and (1.2b) in the FL theory are based on the Pitaevskii-Landau relations - the three identities for the derivatives of the Green's function: $\frac{1,5}{15}$

$$
\begin{aligned}
& \frac{\partial G_{p}^{-1}}{\partial \omega}=\frac{1}{Z}=1-\frac{i}{2} \sum_{\alpha \beta} \int \Gamma_{\alpha \beta, \alpha \beta}^{\omega}\left(p_{F}, q\right)\left(G_{q}^{2}\right)_{\omega} \frac{d^{D+1} q}{(2 \pi)^{D+1}} \\
& \mathbf{p}_{F} \frac{\partial G_{p}^{-1}}{\partial \mathbf{p}}=-\frac{p_{F}^{2}}{m^{*} Z}=-\frac{p_{F}^{2}}{m}+\frac{i}{2} \sum_{\alpha \beta} \int \Gamma_{\alpha \beta, \alpha \beta}^{k}\left(p_{F}, q\right) \frac{\mathbf{p}_{F} \cdot \mathbf{q}}{m}\left(G_{q}^{2}\right)_{k} \frac{d^{D+1} q}{(2 \pi)^{D+1}} \\
& \frac{1}{Z}=1-\frac{i}{2} \sum_{\alpha \beta} \int \Gamma_{\alpha \beta, \alpha \beta}^{\omega}\left(p_{F}, q\right)\left(G_{q}^{2}\right)_{\omega} \frac{\mathbf{p}_{F} \cdot \mathbf{q}}{p_{F}^{2}} \frac{d^{D+1} q}{(2 \pi)^{D+1}} .
\end{aligned}
$$


The first two relations originate from particle-number conservation, while the third relation is a consequence of Galilean invariance. In Eq. (2.2), the object $\left(G_{q}^{2}\right)_{k}$ is the product of two Green's functions with the same frequencies and infinitesimally close momenta, and $\Gamma_{\alpha \beta, \gamma \delta}^{k}$ is the vertex in the limit of zero frequency transfer and vanishing momentum transfer. The latter is related to $\Gamma_{\alpha \beta, \gamma \delta}^{\omega}$ by an integral equation

$$
\begin{aligned}
& \Gamma_{\alpha \beta, \alpha \beta}^{k}(p, q)=\Gamma_{\alpha \beta, \alpha \beta}^{\omega}(p, q) \\
& -\frac{k_{F}^{D-1} Z^{2}}{v_{F}(2 \pi)^{D}} \sum_{\xi, \eta} \int \Gamma_{\alpha \xi, \alpha \eta}^{\omega}\left(p, q^{\prime}\right) \Gamma_{\eta \beta, \xi \beta}^{k}\left(q^{\prime}, q\right) d \Omega_{q}(2.4)
\end{aligned}
$$

In addition, $\left(G_{q}^{2}\right)_{k}$ is related to $\left(G_{q}^{2}\right)_{\omega}$ by

$$
\left(G_{q}^{2}\right)_{k}-\left(G_{q}^{2}\right)_{\omega} \equiv \delta G_{q}^{2}=-\frac{2 \pi i Z^{2} m^{*}}{p_{F}} \delta(\omega) \delta\left(|\mathbf{q}|-p_{F}\right)
$$

Note that Eqs. (2.1) and (2.2) contain the integrals over all intermediate states with momenta $q$. However, using the additional property of Galilean invariance (2.3), one can eliminate the high-energy contribution to $m^{*}$ (but not to $Z$ ). Indeed, substituting Eqs. (2.4) and (2.5) into Eq. (2.2) and using Eq. (2.3), one reduces Eq. (2.2) to

$$
\begin{aligned}
& \mathbf{p}_{F} \frac{\partial G_{p}^{-1}}{\partial \mathbf{p}}=-\frac{p_{F}^{2}}{m^{*} Z}=-\frac{p_{F}^{2}}{m Z}+\frac{i}{2} \frac{p_{F}^{2}}{m^{*} Z} \\
& \times \sum_{\alpha \beta} \int \Gamma_{\alpha \beta, \alpha \beta}^{\omega}\left(p_{F}, q\right) \delta G_{q}^{2} \frac{\mathbf{p}_{F} \cdot \mathbf{q}}{p_{F}^{2}} \frac{d^{D+1} q}{(2 \pi)^{D+1}} \\
& =-\frac{p_{F}^{2}}{m Z}+\frac{p_{F}^{2}}{Z} A_{D} \sum_{\alpha \beta} \int \Gamma_{\alpha \beta, \alpha \beta}^{\omega}\left(p_{F}, q_{F}\right) \frac{\mathbf{p}_{F} \cdot \mathbf{q}_{F}}{p_{F}^{2}} d \Omega_{q},
\end{aligned}
$$

which is equivalent to Eq. (1.2b) for mass renormalization. We emphasize again that Eq. (1.2b), which involves only low-energy fermions, is based not only on particlenumber conservation [Eqs. (2.4) and (2.5)], but also on Eq. (2.3), specific only for Gallilean-invariant systems.

Combining Eqs. (2.1), (2.6), and (2.3), one can construct the self-energy to first order in $\omega$ and $\epsilon_{\mathbf{p}}$ as

$$
\Sigma_{\mathrm{FL}}\left(\omega, \epsilon_{\mathbf{p}}\right)=\left(\omega-\epsilon_{\mathbf{p}}\right)\left[-\frac{i}{2} \sum_{\alpha \beta} \int \Gamma_{\alpha \beta, \alpha \beta}^{\omega}\left(p_{F}, q\right) G_{q}^{2} \frac{d^{D+1} q}{(2 \pi)^{D+1}}\right]+\epsilon_{\mathbf{p}}\left[\frac{i}{2 Z} \sum_{\alpha \beta} \int \Gamma_{\alpha \beta, \alpha \beta}^{\omega}\left(p_{F}, q\right) \frac{\mathbf{p}_{F} \cdot \mathbf{q}}{p_{F}^{2}} \delta G_{q}^{2} \frac{d^{D+1} q}{(2 \pi)^{D+1}}\right] .
$$

\section{B. Perturbation theory for $\Gamma^{\omega}$}

The vertex $\Gamma^{\omega}$ can be obtained via a perturbative expansion in $U(|\mathbf{k}|)$. Diagrams for $\Gamma^{\omega}$ to second order in $U(|\mathbf{k}|)$ are presented in Fig. 1. Assume first that $U(|\mathbf{k}|)=$ const $\equiv U$ (contact interaction). In this case,

$\Gamma_{\alpha \beta, \gamma \delta}^{\omega}\left(p_{F}, q\right)=\delta_{\alpha \gamma} \delta_{\beta \delta}\left[U+i U^{2} \int\left(G_{l} G_{q-p_{F}+l}+G_{l} G_{q+p_{F}-l}\right) \frac{d^{D+1} l}{(2 \pi)^{D+1}}\right]-\delta_{\alpha \delta} \delta_{\beta \gamma}\left[U+i U^{2} \int G_{l} G_{q+p_{F}-l} \frac{d^{D+1} l}{(2 \pi)^{D+1}}\right]$

The first term in Eq. (2.8) is the renormalized interaction with zero momentum transfer, the second term is obtained by antisymmetrization. We see that the first ("direct") term contains contributions from both the particle-hole and particle-particle channels, while the second ("exchange") term contains only a contribution from the particle-particle channel.

In $3 \mathrm{D}$, explicit expression for $\Gamma^{\omega}$ when both particles are on the FS (i.e., $q=q_{F}$ ) were obtained long time ago (see Refs. 1, 6). A similar calculation, performed in
Ref. 12 for the 2D case, yields

$$
\begin{aligned}
& \Gamma_{\alpha \beta, \gamma \delta}^{\omega}\left(p_{F}, q_{F}\right)=\Gamma_{\alpha \beta, \gamma \delta}^{\omega}(\theta) \\
& =\frac{1}{2} \delta_{\alpha \gamma} \delta_{\beta \delta}\left[U+\frac{U^{2} m}{\pi}+\frac{U^{2} m}{2 \pi} \ln \left(\cos \frac{\theta}{2}\right)\right] \\
& -\frac{1}{2} \boldsymbol{\sigma}_{\alpha \gamma} \cdot \boldsymbol{\sigma}_{\beta \delta}\left[U+\frac{U^{2} m}{2 \pi} \ln \left(\cos \frac{\theta}{2}\right)\right]
\end{aligned}
$$

where $\theta$ is the angle between $\mathbf{p}_{F}$ and $\mathbf{q}_{F}$. In deriving Eq. (2.9), we used a result for the static particle-particle 


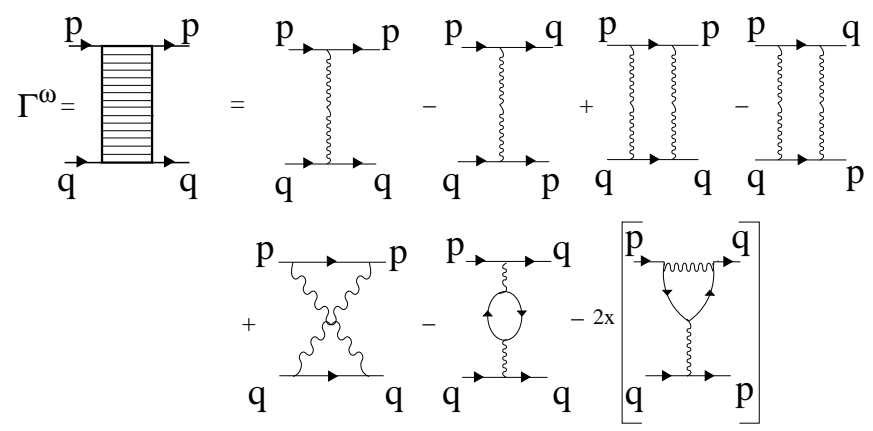

FIG. 1: First and second order diagrams for the Fermi-liquid vertex $\Gamma_{\alpha \beta, \gamma \delta}^{\omega}(p, q)$. The initial four-momenta $p$ and $q$ are associated with spin projections $\alpha$ and $\beta$, respectively. The final four-momenta $p$ and $q$ are associated with spin projections $\gamma$ and $\delta$, respectively.

bubble in $2 \mathrm{D}$

$$
\begin{aligned}
\Pi_{\mathrm{pp}}\left(\omega=0,|\mathbf{k}| \leq 2 p_{F}\right) & =i \int \frac{d^{3} l}{(2 \pi)^{3}} G_{l} G_{k-l} \\
& =\frac{m}{2 \pi} \ln \frac{2 p_{F}}{|\mathbf{k}|}
\end{aligned}
$$

(up to an irrelevant constant). In 2D, the angular dependence of $\Gamma^{\omega}(\theta)$, which is responsible for mass renormalization, comes entirely from the interaction in the particle-particle channel. Since the 2D particle-hole bubble $\Pi_{\mathrm{ph}}(k)=i \int d^{3} l G_{l} G_{l+k} /(2 \pi)^{3}$ is independent of $|\mathbf{k}|$ for $|\mathbf{k}| \leq 2 p_{F}$, renormalization of the interaction in the particle-hole channel only adds a constant to $U$ and is, therefore, irrelevant for $m^{*}$. The formula for $\Gamma_{\alpha \beta, \gamma \delta}^{\omega}\left(p_{F}, q\right)$ for $q$ is away from the FS is rather complex, and we refrain from presenting it.

\section{Effective mass and quasiparticle residue}

Substituting $\Gamma^{\omega}$ from Eq. (2.8) into Eqs. (1.2a) and $(1.2 \mathrm{~b})$ and evaluating the integrals in $3 \mathrm{D}$, we reproduce the known results for $m^{*} / m$ (Refs. 66 7)

$$
\frac{m^{*}}{m}=1+\left(\frac{8}{15}\right)(7 \ln 2-1)\left(\frac{m U p_{F}}{4 \pi^{2}}\right)^{2}
$$

and $Z$ (Ref. 7)

$$
Z=1-8 \ln 2\left(\frac{m U p_{F}}{4 \pi^{2}}\right)^{2} .
$$

In 2D, Eq. (2.9) immediately gives 12

$$
\frac{m^{*}}{m}=1+\frac{1}{2}\left(\frac{m U}{2 \pi}\right)^{2}
$$

while for $Z$ we obtain after numerical integration of $\Gamma_{\alpha \beta, \gamma \delta}^{\omega}\left(p_{F}, q\right)$ instead of (2.9)

$$
Z \approx 1-C\left(\frac{m U}{2 \pi}\right)^{2}
$$

where $C=0.6931 \ldots$ To high numerical accuracy, $C$ is equal to $\ln 2$, but we did not attempt to prove this analytically. We remind that, in the FL formulation, $m^{*} / m$ comes exclusively from the interaction between particles on the FS.

\section{Momentum-dependent interaction}

For a momentum-dependent interaction $U(|\mathbf{k}|)$, expressions for $m^{*} / m$ and $Z$ are generally more complex. Mass renormalization now occurs already at the first order in $U(|q|)$. In 3D,

$$
\frac{m^{*}}{m}=1-\frac{m p_{F}}{16 \pi^{2}}
$$

$\times \int_{0}^{2} d z z\left[U\left(p_{F}(2-z)^{1 / 2}\right)-U\left(p_{F}(2+z)^{1 / 2}\right)\right]+O\left(U^{2}\right)$.

Renormalization of $Z$ still occurs beginning from the second order in $U(|\mathbf{k}|)$.

\section{PERTURBATION THEORY FOR THE SELF-ENERGY}

We now discuss how $m^{*} / m$ and $Z$ occur in the diagrammatic perturbation theory. Again, we consider first the case of a constant interaction $U$; a momentumdependent interaction will be discussed later. The firstorder term in the self-energy (Fig. 2 $a$ ) is irrelevant in this case, and we focus on the second-order diagrams in Fig. 2 Diagram $b$ just shifts the chemical potential and is also irrelevant, so we need to consider only diagrams $c$ and $d$. Relabeling the fermionic momenta, it is easy to see that, for a constant $U$, diagram $d$ is equal to $-1 / 2$ of diagram $c$, so there is essentially one secondorder diagram to be considered, e.g., diagram $c$. This diagram contains three Green's functions, two of which share a common internal momentum. Labeling the momenta as shown in diagram $c$ and integrating over the internal four-momentum $l$, we end up with a particle-hole bubble. Alternatively, labeling the momenta as shown in diagram $e$ and integrating over $l$, we end up with a particle-particle bubble.

We start with combining two internal fermions into a particle-hole bubble; the particle-particle combination is discussed in Sec. IIB 2, Subtracting from the particlehole form of $\Sigma\left(\omega, \epsilon_{\mathbf{p}}\right)$ its value at $\omega=0, \epsilon_{\mathbf{p}}=0$, we find

$$
\Sigma_{\text {pert }}\left(\omega, \epsilon_{\mathbf{p}}\right)-\Sigma(0,0)=-U^{2} \int G_{l} G_{k-p_{F}+l}\left(G_{k+\epsilon}-G_{k}\right) d_{l k},
$$

where $d_{l k} \equiv d^{D+1} l d^{D+1} k /(2 \pi)^{2(D+1)}$ and

$$
\epsilon=\left(\omega, \frac{\epsilon_{\mathbf{p}}}{v_{F} p_{F}} \mathbf{p}_{F}\right)
$$


a)

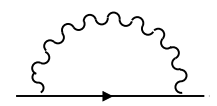

b)

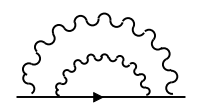

c)

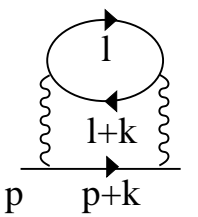

d)

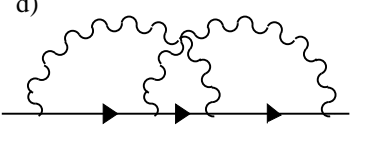

e)

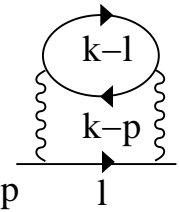

FIG. 2: First and second order diagrams for the fermionic self-energy $\Sigma_{\text {pert }}\left(\omega, \epsilon_{\mathbf{p}}\right)$. For a momentum-indepedent interaction $U(|\mathbf{q}|)=U$, only second- and higher-order diagrams renormalize the mass and $Z$. For a momentum-dependent interaction, mass renormalization starts already at the firstorder. Diagram $e$ is the same as $c$, except for internal fermions are combined into a particle-particle rather than a particlehole pair.

is the (small) external four-momentum. The self-energy can be further split into two parts as

$$
\Sigma_{\text {pert }}\left(\omega, \epsilon_{\mathbf{p}}\right)-\Sigma(0,0)=\delta \Sigma_{1}\left(\omega, \epsilon_{\mathbf{p}}\right)+\delta \Sigma_{2}\left(\omega, \epsilon_{\mathbf{p}}\right),
$$

where

$$
\begin{aligned}
& \delta \Sigma_{1}\left(\omega, \epsilon_{\mathbf{p}}\right)=U^{2} \int G_{l} G_{k-p_{F}+l} G_{q}^{2}\left(\omega-\epsilon_{\mathbf{p}} \frac{\mathbf{p}_{F} \cdot \mathbf{k}}{p_{F}^{2}}\right) d_{l k} \\
& \delta \Sigma_{2}\left(\omega, \epsilon_{\mathbf{p}}\right)=-U^{2} \int^{\prime} G_{l} G_{k-p_{F}+l}\left(G_{k-\epsilon}-G_{k}\right) d_{l k} .
\end{aligned}
$$

The difference between the two parts is as follows. In the first part, the integrand was expanded to first order in $\omega$ and $\epsilon_{\mathbf{p}}$. This is justified if typical internal energies remain finite when $\omega, \epsilon_{\mathbf{p}} \rightarrow 0$. This is a regular, high-energy contribution to the self-energy coming from fermions not confined to the FS. The second term is an anomalous contribution from internal energies of order $\omega$ and $\epsilon_{\mathbf{p}}$, which cannot be obtained by an expansion of $\Sigma_{\text {pert }}$ in the external energies. This second term is a low-energy contribution (to emphasize this, we put a prime on the integral for this part).

\section{A. 3D case}

Evaluating the integrals in Eqs. 3.4a and 3.4b for the $3 \mathrm{D}$ case, we find that both contributions are finite; namely

$$
\begin{aligned}
\delta \Sigma_{1}\left(\omega, \epsilon_{\mathbf{p}}\right)= & 8 \ln 2\left(\frac{m U p_{F}}{4 \pi^{2}}\right)^{2}\left(\omega-\epsilon_{\mathbf{p}}\right) \\
& +\frac{4}{3} \epsilon_{\mathbf{p}}(4 \ln 2-1)\left(\frac{m U p_{F}}{4 \pi^{2}}\right)^{2} \\
\delta \Sigma_{2}\left(\omega, \epsilon_{\mathbf{p}}\right)= & -\frac{4}{5} \epsilon_{\mathbf{p}}(2 \ln 2-1)\left(\frac{m U p_{F}}{4 \pi^{2}}\right)^{2} .
\end{aligned}
$$

Adding up two parts and casting the result into the form of Eq. (1.3), we recover the Galitskii's result (Ref. 7):

$$
\begin{aligned}
\Sigma_{\text {pert }}\left(\omega, \epsilon_{\mathbf{p}}\right)- & \Sigma(0,0)=\left(\frac{m U p_{F}}{4 \pi^{2}}\right)^{2} \\
& \times\left[8 \ln 2\left(\omega-\epsilon_{\mathbf{p}}\right)+\frac{8}{15}(7 \ln 2-1) \epsilon_{\mathbf{p}}\right]
\end{aligned}
$$

This self-energy indeed produces the same $m^{*} / m$ and $Z$ as in the FL theory, Eqs. 2.12) and (2.11), We see, however, that mass renormalization-determined by a standalone $\epsilon_{\mathbf{p}}$ term in the self-energy -comes from both the high- and low-energy parts of $\Sigma_{\text {pert }}$. Only the sum of the two contributions recovers the FL formula for $m^{*} / m$. On the other hand, renormalization of $Z$ comes only from $\delta \Sigma_{1}$, i.e., from high energies.

\section{B. 2D case}

The difference between the FL and diagrammatic approaches becomes even more obvious in $2 \mathrm{D}$. Since, to the best of our knowledge, the $Z$ factor for a $2 \mathrm{D}$ Fermi liquid with a short-range interaction has not been calculated before, we consider the $2 \mathrm{D}$ case in more detail. We also use the $2 \mathrm{D}$ case as an example to show that an interplay between high-energy and low-energy contributions to the effective mass (but not the full result) depends on whether the self-energy is calculated via particle-hole or particle-particle bubbles.

\section{1. perturbative self-energy via particle-hole bubble}

The calculation of the perturbative self-energy via a particle-hole bubble is based on Eqs. (3.4a and (3.4b). It $2 \mathrm{D}$, the particle-hole bubble can be found analytically for any $\omega$ and $|\mathbf{k}|$ : 


$$
\Pi_{\mathrm{ph}}(\omega, \mathbf{k})=-\frac{m}{2 \pi}\left[1+i \frac{\sqrt{2} \tilde{\omega}}{\sqrt{\tilde{k}^{2}-\tilde{k}^{4}-\tilde{\omega}^{2}+\sqrt{\left(\tilde{k}^{2}-\tilde{k}^{4}-\tilde{\omega}^{2}\right)^{2}-4 \tilde{\omega}^{2} \tilde{k}^{4}}}}\right]
$$

where $\tilde{\omega}=2 \omega m / p_{F}^{2}$ and $\tilde{k}=|\mathbf{k}| / 2 p_{F}$. To calculate the regular part of the self-energy, one needs to know the entire bubble, while the anomalous part is determined only by the static bubble $\Pi_{\mathrm{ph}}(0,|\mathbf{k}|)$. Performing the angular integral in $\delta \Sigma_{1}$ analytically and remaining integrals numerically, and all integrals in $\delta \Sigma_{2}$ analytically, we obtain

$$
\begin{aligned}
& \delta \Sigma_{1}\left(\omega, \epsilon_{\mathbf{p}}\right)=C\left(\frac{m U}{2 \pi}\right)^{2}\left(\omega-\epsilon_{\mathbf{p}}\right)+\frac{\epsilon_{\mathbf{p}}}{2}\left(\frac{m U}{2 \pi}\right)^{2}(3.8 \mathrm{a}) \\
& \delta \Sigma_{2}\left(\omega, \epsilon_{\mathbf{p}}\right)=0,
\end{aligned}
$$

with

$$
C=\frac{1}{2 \pi} \int_{0}^{\infty} d x \int_{0}^{\infty} d y \frac{\partial}{\partial y} \frac{\sqrt{A+\sqrt{A^{2}+x^{2} y^{2}}}}{\sqrt{A^{2}+x^{2} y^{2}}} F(x, y),
$$

$A=\left(x-x^{2}+y^{2}\right) / 2$, and

$$
F(x, y)=\frac{y}{\sqrt{A+\sqrt{A^{2}+x^{2} y^{2}}}}-\theta(x-1) \sqrt{\frac{x-1}{x}}(3 .
$$

Numerical integration yields, to high accuracy, $C=$ $0.6931 \cdots=\ln 2$; same as for the prefactor in Eq. (2.14).

The reason why $\delta \Sigma_{2}=0$ in $2 \mathrm{D}$ is very simple. This contribution is expressed via a static particle-hole bubble as

$$
\begin{aligned}
\delta \Sigma_{2}\left(\omega, \epsilon_{\mathbf{p}}\right)= & i \frac{\epsilon_{\mathbf{p}} U^{2}}{2 \pi^{2} v_{F}} \int_{0}^{2 p_{F}} d|\mathbf{k}| \Pi_{\mathrm{ph}}(\omega=0,|\mathbf{k}|) \\
& \times \frac{1-|\mathbf{k}|^{2} / 2 p_{F}^{2}}{\sqrt{1-\left(|\mathbf{k}| / 2 p_{F}\right)^{2}}}
\end{aligned}
$$

As $\Pi_{\mathrm{ph}}(\omega=0,|\mathbf{k}|)$ is independent of $|\mathbf{k}|$ for $\mathbf{k} \mid \leq 2 p_{F}$, the integral over $|\mathbf{k}|$ vanishes. For the same reason, mass renormalization in the FL theory comes only from the particle-particle part of the vertex in Eq. (2.9).

Casting the result into the form of Eq. (1.3), we again reproduce the FL results for $m^{*} / m$ and $Z$, Eqs. (2.13) and (2.14). However, we see that now $m^{*} / m$ comes solely from the high-energy part of the self-energy, in an (apparent) contradiction to the FL theory, where it comes from low energies.

\section{2. perturbative self-energy via particle-particle bubble}

We now show that an interplay between high- and lowenergy contributions to the perturbative self-energy depends on the way how we obtain it. To demonstrate this, we obtain the same perturbative self-energy $\Sigma_{\text {pert }}\left(\omega, \epsilon_{p}\right)$ as in Eq. (3.8a by combining internal fermions into particle-particle rather than particle-hole pairs. We will see that in this situation mass renormalization comes from both high- and low energies.

For simplicity, we consider a momentum-independent interaction in $2 \mathrm{D}$ and restrict attention to the secondorder in $U$. Labeling the momenta as shown in diagram $e$ of Fig. 2, we obtain for the second-order self-energy

$\tilde{\Sigma}_{\text {pert }}\left(\omega, \epsilon_{\mathbf{p}}\right)-\tilde{\Sigma}(0,0)=-U^{2} \int G_{l} G_{k+p_{F}-l}\left(G_{k-\epsilon}-G_{k}\right) d_{l k}$.

We denote the self-energy obtained in this way as $\tilde{\Sigma}_{\text {pert }}$ to distinguish it from the self-energy in the particle-hole form. As before, we split the difference $\tilde{\Sigma}_{\text {pert }}\left(\omega, \epsilon_{\mathbf{p}}\right)-$ $\tilde{\Sigma}(0,0)$ into a sum of regular and anomalous contributions as

$$
\delta \tilde{\Sigma}_{1}\left(\omega, \epsilon_{\mathbf{p}}\right)=U^{2} \int G_{l} G_{k+p_{F}-l} G_{k}^{2}\left(\omega-\epsilon_{\mathbf{p}} \frac{\mathbf{p}_{F} \cdot \mathbf{k}}{p_{F}^{2}}\right) d_{l k}
$$

$$
\delta \tilde{\Sigma}_{2}\left(\omega, \epsilon_{\mathbf{p}}\right)=-U^{2} \int^{\prime} G_{l} G_{k+p_{F}-l}\left(G_{k-\epsilon}-G_{k}\right) d_{l k} .
$$

The anomalous part $\delta \tilde{\Sigma}_{2}$ is now expressed via a static particle-particle bubble as

$$
\begin{aligned}
\delta \tilde{\Sigma}_{2}\left(\omega, \epsilon_{\mathbf{p}}\right)= & -\frac{\epsilon_{\mathbf{p}} U^{2}}{2 \pi^{2} v_{F}} \int_{0}^{2 p_{F}} d|\mathbf{k}| \Pi_{\mathrm{pp}}(\omega=0,|\mathbf{k}|) \\
& \times \frac{1-|\mathbf{k}|^{2} / 2 p_{F}^{2}}{\sqrt{1-\left(|\mathbf{k}| / 2 p_{F}\right)^{2}}}
\end{aligned}
$$

where $\Pi_{\mathrm{pp}}(\omega=0,|\mathbf{k}|)$ is given in Eq. (2.10). In contrast to Eq. (3.11), the integral over $|\mathbf{k}|$ now does not vanish, and we obtain

$$
\delta \tilde{\Sigma}_{2}\left(\omega, \epsilon_{\mathbf{p}}\right)=-\frac{\epsilon_{\mathbf{p}}}{2}\left(\frac{m U}{2 \pi}\right)^{2} .
$$

Therefore, in contrast to the particle-hole case, the low-energy contribution to mass renormalization in the 
particle-particle case is finite but opposite in sign to mass renormalization in the FL theory, Eq. (2.13).

The regular part of self-energy, $\delta \tilde{\Sigma}_{1}$, renormalizes both the $Z$ factor and effective mass. To evaluate this part of the self-energy, a static approximation for $\Pi_{p p}$ is not sufficient, and we need a dynamic form of the particleparticle propagator. Re-expressing $\delta \tilde{\Sigma}_{1}$ in 3.13a via the particle-particle polarization bubble and shifting the momentum as $k-p_{F} \rightarrow k$, we obtain

$$
\begin{aligned}
& \delta \tilde{\Sigma}_{1}\left(\omega, \epsilon_{\mathbf{p}}\right)=U^{2} \int \frac{\Pi_{p p}\left(\omega_{k}, \mathbf{k}\right)}{\left(\omega_{k}-\epsilon_{k-p_{F}}\right)^{2}} \\
& \times\left[\left(\omega-\epsilon_{\mathbf{p}}\right)+\epsilon_{\mathbf{p}}\left(2-\frac{\mathbf{p}_{F} \cdot \mathbf{k}}{p_{F}^{2}}\right)\right] d_{k} .
\end{aligned}
$$

We remind that $d_{k}=k d k d \theta_{k} d \omega_{k} /(2 \pi)^{3}$. If we approximated $\Pi_{p p}\left(\omega_{k}, \mathbf{k}\right)$ by its satic form, $\delta \tilde{\Sigma}_{1}$ would vanish after integration over $\omega_{k}$ because of the double pole. The dynamic $\Pi_{p p}\left(\omega_{k}, \mathbf{k}\right)$, however, has branch cuts in both upper and lower half-planes of $\omega_{k}$, which ensures that the frequency integral is non-zero.

The dynamic polarization bubble is obtained by standard means and, for $|\mathbf{k}|<2 p_{F}$, is given by

$$
\Pi_{\mathrm{p} p}\left(\omega_{k}, \mathbf{k}\right)=\frac{m}{2 \pi^{2}} \int_{0}^{\pi / 2} d \phi \ln S_{\phi}\left(\omega_{k}, \mathbf{k}\right)
$$

where

$$
\begin{aligned}
& S_{\phi}\left(\omega_{k}, \mathbf{k}\right)=\frac{m \omega_{k}-p_{F}^{2}+\mathbf{k}^{2} / 4}{m \omega_{k}-|\mathbf{k}| \cos \phi \sqrt{p_{F}^{2}+\frac{\mathbf{k}^{2}}{4} \sin ^{2} \phi}-\frac{\mathbf{k}^{2}}{2} \cos ^{2} \phi} \\
& \times \frac{p_{F}^{2}}{m \omega_{k}+|\mathbf{k}| \cos \phi \sqrt{p_{F}^{2}-\frac{\mathbf{k}^{2}}{4} \sin ^{2} \phi}+\frac{\mathbf{k}^{2}}{2} \cos ^{2} \phi}
\end{aligned}
$$

For $|\mathbf{k}|>2 p_{F}$ the expression for $\Pi_{p p}$ is more complex, but we do not need it as in this region the pole and branch cut are in the same half-plane of $\omega_{k}$ and the frequency integral vanishes.

Substituting (3.17) into (3.16), evaluating the frequency integral over a half plane where there is no double pole (this requires separate considerations for $|\mathbf{k}|>$ $2 p_{F} \cos \theta_{k}$ and $\left.|\mathbf{k}|<2 p_{F} \cos \theta_{k}\right)$, and evaluating the remaining integrals over $\phi, \theta_{k}$ and $|\mathbf{k}|$ numerically, we obtain

$$
\delta \tilde{\Sigma}_{1}\left(\omega, \epsilon_{\mathbf{p}}\right)=C\left(\frac{m U}{2 \pi}\right)^{2}\left(\omega-\epsilon_{\mathbf{p}}\right)+\epsilon_{\mathbf{p}}\left(\frac{m U}{2 \pi}\right)^{2} .
$$

where, as before, $C=0.6931 \cdots=\ln 2$.

The total particle-particle self-energy, given by the sum of Eqs. (3.15) and (3.19), is indeed the same as the total particle-hole self-energy, given by the sum of Eqs. (3.8a) and (3.8b), and total mass renormalization is the same as in Eq. 2.13). However, we see that mass renormalization now comes from both high and low energies.

\section{Momentum-dependent interaction}

For a momentum-dependent interaction $U(|\mathbf{k}|)$, the difference between the diagrammatic and FL formulas for the self-energy is less drastic. In particular, for a weak but momentum-dependent interaction, mass renormalization to first order in $U(|\mathbf{k}|)$, as defined by Eq. (2.15), comes only from fermions on the FS.

For completeness, we also present perturbative results for $m^{*} / m$ and $Z$ for the Coulomb interaction. At small $r_{s}$, both $m^{*} / m$ and $Z$ are quasi-linear in $r_{s}$ for small $r_{s}$. In 3D, $m^{*} / m=1-\left(r_{s} / 2 \pi\right)(4 / 9 \pi)^{1 / 3} \ln r_{s}^{-1}$ and $Z=1-$ $0.17696 r_{s}$ (Ref. 10). In 2D, $m^{*} / m=1-\left(r_{s} / \pi \sqrt{2}\right) \ln r_{s}^{-1}$ (Ref. 10) and $Z=1-\left(r_{s} / \sqrt{2}\right)(1 / 2+1 / \pi)$ (Ref. 11). The case of Coulomb interaction is special in that $Z$ comes from fermions in the vicinity of the FS.

\section{RECONCILIATION OF THE FERMI-LIQUID AND PERTURBATIVE APPROACHES}

We have shown in the previous sections that while mass renormalization comes from low energies in the FL theory, it generally contains both high- and low-energy contributions in a diagrammatic perturbation theory. In Secs. IV A IV C, we show how to reconcile the two approaches.

\section{A. Equivalence of the Fermi-liquid and perturbative aprpoaches for a momentum-independent interaction}

To begin, we emphasize that the results for the selfenergy in the FL and perturbative approaches need not to coincide identically, because $\Sigma_{\mathrm{FL}}$ given by Eq. (2.7) is only an expansion of the full self-energy to first order in $\omega$ and $\epsilon_{\mathbf{p}}$, while $\Sigma_{\text {pert }}$ given by Eqs. 3.313.4a and (3.4b contains all orders in $\omega$ and $\epsilon_{\mathbf{p}}$. However, $\Sigma_{\text {pert }}$ to first order in $\omega$ and $\epsilon_{\mathbf{p}}$ must coincide with $\Sigma_{\mathrm{FL}}$.

Comparing the two self-energies, we see the difference: while $\Sigma_{\mathrm{FL}}$, expressed via $\Gamma^{\omega}$, contains both particlehole and particles-particle bubbles, $\Sigma_{\text {pert }}$ contains only a particle-hole bubble. We now show that there exists a particular relation between the combinations of the Green's functions which involve particle-hole and particle-particle bubbles, namely

$$
\int\left(G_{l} G_{k-p_{F}+l}+G_{l} G_{k+p_{F}-l}\right)\left(G_{k-\epsilon}-G_{k}\right) d_{k l}=0
$$

where $\epsilon_{\mathbf{p}}$ is given by Eq. (3.2). This relation is valid to first order in $\omega$ and $\epsilon_{\mathbf{p}}$ and follows from the identity, which we have already used implicitly when $\operatorname{diagram} c$ in 
Fig. 2 was replaced by diagram $e$ :

$$
\begin{aligned}
& \int d_{k l} G_{l} G_{k-p_{F}+l}\left(G_{k+\epsilon}-G_{k}\right) \\
& =\int d_{k l} G_{l} G_{k+p_{F}-l}\left(G_{k-\epsilon}-G_{k}\right) .
\end{aligned}
$$

The identity in Eq. (4.2) is proven by relabeling the fourmomenta, e.g., by relabeling the momenta in both terms in the second line as $k \rightarrow k-p_{F}+l+\epsilon$, and then relabeling $k+\epsilon \rightarrow k$ in the last term. Equation (4.1) follows from (4.2) once $G_{k+\epsilon}-G_{k}$ in the first line of Eq. (4.2) is replaced by $G_{k}-G_{k-\epsilon}+\mathcal{O}\left(\epsilon^{2}\right)$. Adding Eq.
(4.1) to $\Sigma_{\text {pert }}\left(\omega, \epsilon_{\mathbf{p}}\right)$, we find, after simple algebra, that it becomes equal to $\Sigma_{\mathrm{FL}}$, i.e., the expressions for $m^{*} / m$ and $Z$ become exactly the same as in Fermi liquid theory. Analyzing further the left-hand side of Eq. (4.1), we find that both the particle-particle and particle-hole terms contain regular (high-energy) and anomalous (lowenergy) contributions. Anomalous contributions contain only the single-particle dispersion $\epsilon_{\mathbf{p}}$, while regular contributions contain both $\epsilon_{\mathbf{p}}$ and $\omega$ terms. Expanding the regular contributions to first order in $\omega$ and $\epsilon_{\mathbf{p}}$ and equating the prefactors of $\epsilon_{\mathbf{p}}$ and $\omega$ terms, we obtain

$$
\begin{gathered}
\omega \int d_{k l}\left(G_{l} G_{k-p_{F}+l}+G_{l} G_{k+p_{F}-l}\right) G_{k}^{2}=0 \\
\epsilon_{\mathbf{p}} \int d_{k l}\left(G_{l} G_{k-p_{F}+l}+G_{l} G_{k+p_{F}-l}\right) G_{k}^{2} \frac{\mathbf{p}_{F} \cdot \mathbf{k}}{p_{F}^{2}}=-\epsilon_{\mathbf{p}} \int d_{k l}\left(G_{l} G_{k-p_{F}+l}+G_{l} G_{k+p_{F}-l}\right) \delta G_{k}^{2} \frac{\mathbf{p}_{F} \cdot \mathbf{k}}{p_{F}^{2}}
\end{gathered}
$$

where $\delta G_{k}^{2}$, defined by Eq. (2.5), projects the integral over $k$ onto the FS. The left- and right-hand sides of Eq. (4.4) are anomalous (low-energy) and regular (highenergy) contributions, respectively.

We see from Eq. (4.3) that the addition of Eq. (4.1) to $\Sigma_{\text {pert }}$ does not change the result for the $\omega$ term in a sense that there is no interplay between high-and lowenergy contributions, and Eq. (4.3) simply adds zero to the high-energy contribution. This explains why the $\omega$ terms in $\Sigma_{\mathrm{FL}}$ and $\Sigma_{\text {pert }}$ are identical. On the other hand, by adding Eq. (4.1) to $\Sigma_{\text {pert }}$ we are changing the interplay between the high- and low-energy contributions to the $\epsilon_{\mathbf{p}}$ terms. The regular contribution from Eq. (4.4) cancels the $\epsilon_{\mathbf{p}}$ term in $\delta \Sigma_{1}$, while the anomalous contribution renders $\delta \Sigma_{2}$ equal to the FL self-energy, Eq. (2.7).

\section{B. Momentum-dependent interaction}

The results of the previous section can be readily extended to the case of a momentum-dependent interaction. In this situation, we obtain, instead of Eqs. (2.813.4a) and (3.4b)

$$
\begin{aligned}
& \Gamma_{\alpha \beta, \alpha \beta}^{\omega}\left(p_{F}, q\right)=U(0)+i \int \frac{d^{D+1} l}{(2 \pi)^{D+1}} U^{2}\left(\left|\mathbf{p}_{F}-\mathbf{l}\right|\right)\left(G_{l} G_{q-p_{F}+l}+G_{l} G_{q+p_{F}-l}\right) \\
& -\delta_{\alpha \beta}\left[U\left(\left|\mathbf{q}-\mathbf{p}_{F}\right|\right)-i \int \frac{d^{D+1} l}{(2 \pi)^{D+1}}\left[\left\{2 U\left(\left|\mathbf{q}-\mathbf{p}_{F}\right|\right)-2 U\left(\left|\mathbf{q}-\mathbf{p}_{F}\right|\right) U\left(\left|\mathbf{p}_{F}-\mathbf{l}\right|\right)\right\} G_{l} G_{l+q-p_{F}}-U\left(\left|\mathbf{p}_{F}-\mathbf{l}\right|\right) U(|\mathbf{l}-\mathbf{q}|) G_{l} G_{q+p_{F}-l}\right]\right.
\end{aligned}
$$

and $\delta \Sigma_{\text {pert }}\left(\omega, \epsilon_{\mathbf{p}}\right)=\Sigma_{\text {pert }}\left(\omega, \epsilon_{\mathbf{p}}\right)-\Sigma_{\text {pert }}(0,0)=\delta \Sigma_{1}+\delta \Sigma_{2}$ with

$$
\begin{aligned}
\delta \Sigma_{1}\left(\omega, \epsilon_{\mathbf{p}}\right)= & \int d_{l q}\left[2 G_{l} G_{k-p_{F}+l}\left\{U^{2}\left(\left|\mathbf{q}-\mathbf{p}_{F}\right|\right)-2 U\left(\left|\mathbf{p}_{F}-\mathbf{l}\right|\right) U\left(\left|\mathbf{q}-\mathbf{p}_{F}\right|\right)\right\}-G_{l} G_{q+p_{F}-l} U\left(\left|\mathbf{p}_{F}-\mathbf{l}\right|\right) U(|\mathbf{l}-\mathbf{q}|)\right]\left(G_{k-\epsilon}-G_{k}\right) \\
\delta \Sigma_{2}\left(\omega, \epsilon_{\mathbf{p}}\right)= & -\int d_{l q}\left[2 G_{l} G_{k-p_{F}+l}\left\{U^{2}\left(\left|\mathbf{q}-\mathbf{p}_{F}\right|\right)-2 U\left(\left|\mathbf{p}_{F}-\mathbf{l}\right|\right) U\left(\left(\left|\mathbf{q}-\mathbf{p}_{F}\right|\right)\right\}-G_{l} G_{q+p_{F}-l} U\left(\left|\mathbf{p}_{F}-\mathbf{l}\right|\right) U(|\mathbf{l}-\mathbf{q}|)\right] G_{q}^{2}\right. \\
& \times\left(\omega-\epsilon_{\mathbf{p}} \frac{\mathbf{p}_{F} \cdot \mathbf{q}}{p_{F}^{2}}\right)
\end{aligned}
$$

where we neglected first-order terms.
Comparing the expressions for $\Gamma^{\omega}$ and $\delta \Sigma_{\text {pert }}$, we see that $\Gamma^{\omega}$ [and, hence, $\Sigma_{\mathrm{FL}}$ given by Eq. (2.7)] again con- 
tains two extra terms not present in the diagrammatic self-energy. These two terms have the same overall factor of $U^{2}\left(\left|\mathbf{p}_{F}-\mathbf{l}\right|\right)$. After some re-arranging of the momenta in the products of three fermionic propagators, we obtain an analog of Eq. (4.1) for a momentum-dependent interaction as

$$
\int d_{q l} U^{2}\left(\left|\mathbf{p}_{F}-\mathbf{l}\right|\right)\left(G_{l} G_{k-p_{F}+l}+G_{l} G_{q+p_{F}-l}\right)\left(G_{k-\epsilon}-G_{k}\right)
$$

Adding this expression to $\delta \Sigma_{\text {pert }}$, we find after some algebra that high-energy contributions to $m^{*} / m$ cancel and, to first order in $\omega$ and $\epsilon_{\mathbf{p}}, \Sigma_{\text {pert }}$ becomes equal to $\Sigma_{\mathrm{FL}}$.

\section{Higher orders of the perturbation theory}

So far, we have focused only on the lowest-order perturbation theory. One can show, however, that Eq. (4.6) remains valid if the bare fermionic propagators are replaced by the full ones and $U(|\mathbf{q}|)$ is replaced by a fully renormalized interaction which depends not only on the momentum but also on frequency. This is so because Eq. (4.6) is proven simply by re-arranging internal four-momenta. Next, the full perturbative self-energy is also obtained from the second-order result, Eq. (4.5), by dressing the interactions and propagators. Adding Eq. (4.6) to the full perturbative self-energy, expressed via the full propagators and full interactions, we immediately recover $\Sigma_{\mathrm{FL}}$ simply because the previous proof of this statement did not rely on the specific forms of $G$ and $U$.

\section{V. $S U(N)$-INVARIANT FERMI LIQUID}

$$
\text { A. } S U(N) \text { vs } S U(2)
$$

In this section, we discuss the interplay between the FL- and perturbation theories for a system of interacting fermions with a large number of flavors $N$. Fermiliquid properties of such a system were discussed both in terms of $\mathrm{RG}^{13}$ and pertubation theory for the case of a Coloumb interaction. ${ }^{14,15}$ In the limit when the $N$ times a (dimensionless) coupling constant is larger than one, renormalization of not only the $Z$ factor but also of the effective mass comes from energies much higher than the Fermi energy. Based on the observation, the authors of Ref. 14 argued that an $S U(N)$-invariant FL is not of the same type as discussed in the framework of the Landau theory. We show here that this is not the case: the FL- and perturbation theories give the same results for the $S U(N)$-invariant case as well. To see this, however, one needs to collect next-to-leading terms in the large$N$ expansion of the FL theory, whereas the perturbation theory can be evaluated only to the leading order in $1 / N$.

The difference between the large- $N$ expansions for the
FL- and perturbation theories is most dramatic for the case of a momentum-independent interaction in $2 \mathrm{D}$, and for brevity we consider only this case here. In the $S U(N)$ case, each particle-hole bubble is multiplied by a factor of $N$. The second-order self-energy contains two diagrams $-c$ and $d$ in Fig. 2-the first of which acquires a factor of $N$ while the second does not. Consequently, the perturbative effective mass acquires a factor of $N-1$ compared to the result in Eq. (2.13)

$$
\frac{m^{*}}{m}=1+\frac{N-1}{2}\left(\frac{m U}{2 \pi}\right)^{2} \approx 1+\frac{N}{2}\left(\frac{m U}{2 \pi}\right)^{2},
$$

where the last result applies to the large- $N$ limit. On the other hand, the only diagram for $\Gamma^{\omega}$ which acquires a factor of $N$-diagram $f$ in Fig. 1-does not depend on the angle between the initial fermionic momenta $\mathbf{p}_{F}$ and $\mathbf{q}_{F}$, because the static particle-hole bubble is independent of the momentum in $2 \mathrm{D}$ for $\left|\mathbf{p}_{\mathbf{F}}-\mathbf{q}_{\mathbf{F}}\right| \leq 2 p_{F}$. Therefore, the leading term in the $1 / N$ expansion for $\Gamma^{\omega}$ does not contribute to mass renormalization. To resolve this contradiction, one needs to recall that, when deriving the FL result for $m^{*}\left(1.2 \mathrm{~b}\right.$ ) we divided the trace of $\Gamma^{\omega}$ by the number of spin components. This is the origin of the factor of two in the prefactor $A_{D}$. The formula for the $S U(N)$ case is obtained from Eq. 1.2b simply by replacing 2 in $A_{D}$ by $N$; in $2 \mathrm{D}$, we have

$$
\frac{1}{m^{*}}=\frac{1}{m}-\frac{Z^{2}}{N(2 \pi)^{2}} \sum_{\alpha \beta} \int \Gamma_{\alpha \beta, \alpha \beta}^{\omega}\left(p_{F}, q_{F}\right) \frac{\mathbf{p}_{F} \cdot \mathbf{q}_{F}}{p_{F}^{2}} d \Omega_{q} .
$$

Diagrams for "direct" processes $(\mathbf{p} \rightarrow \mathbf{p}, \mathbf{q} \rightarrow \mathbf{q})$ enter $\Gamma^{\omega}$ with a factor of $\delta_{\alpha \gamma} \delta_{\beta, \delta}$, which becomes equal to one for $\gamma=\alpha$ and $\delta=\beta$. Therefore, the trace of the direct contribution to $\Gamma^{\omega}$ gives a factor of $N^{2}$ which, upon dividing by an overall factor of $N$ in Eq. (5.2), gives an $\mathcal{O}(N)$ contribution to $m^{*}$. To obtain an $\mathcal{O}(N)$ term in $m^{*}$ from the FL theory, one thus needs to collect all $d i$ rect $\mathcal{O}(1)$ diagrams for $\Gamma^{\omega}$. On the other hand, diagrams for "exchange" processes $(\mathbf{p} \rightarrow \mathbf{q}, \mathbf{q} \rightarrow \mathbf{p})$ enter $\Gamma^{\omega}$ with a factor of $\delta_{\alpha \delta} \delta_{\beta \gamma}$, which becomes equal to $\delta_{\alpha \beta}$ for $\gamma=\alpha$ and $\delta=\beta$. The trace of the exchange contribution is of order $N$, which translates into a subleading, $\mathcal{O}(1)$ term in $m^{*}$. Therefore, one can neglect exchange processes in $\Gamma^{\omega}$ in the large- $N$ limit. A physical reason for this simplification is obvious: since the large- $N$ limit is inherently semiclassical, the Pauli principle becomes irrelevant. 
The same conclusion also follows from an identity which involves the generators $\hat{T}$ of the $S U(N)$ group 13

$$
\delta_{\alpha \delta} \delta_{\beta \gamma}=\frac{1}{2} \sum_{a=1}^{N^{2}-1} \hat{T}_{\alpha \gamma}^{a} \hat{T}_{\beta \delta}^{a}+\frac{1}{N} \delta_{\alpha \gamma} \delta_{\beta \delta}
$$

The delta-symbols on the left (right) occur in exchange (direct) contributions to $\Gamma^{\omega}$, correspondingly. Since $\hat{T}^{a}$ are traceless, it follows immediately that the trace of the exchange contribution to $\Gamma^{\omega}$ is by a factor of $N$ smaller than the trace of the direct contribution.

Coming back to the second-order $\Gamma^{\omega}$ for arbitrary $N$, we need to consider all subleading, $\mathcal{O}(1)$ diagrams in Fig. (11). Diagrams $e$ and $g$ contain particle-hole bubbles evaluated at $\left|\mathbf{p}_{F}-\mathbf{q}_{F}\right|$ and, therefore, do not contribute to mass renormalization. Direct particle-particle diagram $c$ contributes an $\mathcal{O}(N)$ term to $m^{*}$, while its exchange counterpart $d$ contributes an $\mathcal{O}(1)$. A combined contribution of diagrams $c$ and $d$ is $(N-1)$ times the $S U(2)$ result, which is the same as in Eq. (5.1).

In the $S U(2)$ case, the perturbative regime implies that the interaction is weak in a sense that $m U \ll 1$. In the $S U(N)$ case with a large number of flavors, there is an intermediate range of interactions, defined by the condition $1 / N \ll U m \ll 1$, where the perturbation theory can be resummed to infinite order. It is instructive to compare the perturbation theory with the FL formalism in this case. The subsequent analysis will be performed in the Matsubara technique at $T=0$.

\section{B. Pertubation theory for the self-energy in the regime $1 / N \ll U m \ll 1$}

In the large- $N$ limit, the perturbative self-energy is given diagrams with a maximal number of the particlehole bubbles at each order in $U$. The sum of such diagrams is equivalent to a first-order diagram, shown in Fig. VB.

$\Sigma_{\text {pert }}\left(\omega, \epsilon_{\mathbf{p}}\right)=\int \frac{d^{2} k}{(2 \pi)^{2}} \int \frac{d \omega_{k}}{2 \pi} G\left(\omega+\omega_{k}, \mathbf{p}+\mathbf{k}\right) \tilde{U}\left(\omega_{k}, \mathbf{k}\right)$,

where the effective interaction is of an RPA form

$$
\tilde{U}\left(\omega_{k}, \mathbf{k}\right)=\frac{U}{1-N U \Pi_{\mathrm{ph}}\left(\omega_{k}, \mathbf{k}\right)} .
$$

As in the $S U(2)$ case, the self-energy can be separated into anomalous and regular part. The anomalous part is similar to Eq. (3.11), except for the secondorder effective interaction $U^{2} \Pi_{\mathrm{ph}}\left(\omega_{k}=0, \mathbf{k}\right)$ is replaced by $\tilde{U}\left(\omega_{k}=0, \mathbf{k}\right)$. Still, as $\left.\Pi_{\mathrm{ph}}\left(\omega_{k}=0, \mathbf{k}\right)\right)$ is independent of $|\mathbf{k}|$ for $|\mathbf{k}| \leq 2 p_{F}$, the integral over $|\mathbf{k}|$ vanishes, and the anomalous part of the self-energy does not renormalize the effective mass.

In the regular part of the self-energy, we assume-and then justify-that the limit of $N U m \gg 1$ corresponds to

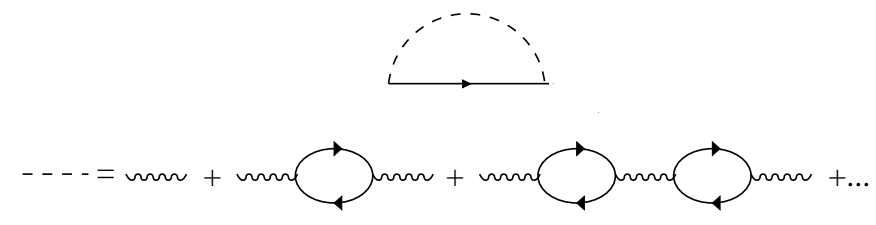

FIG. 3: Self-energy in the large- $N$ limit. The dashed line is an effective interaction given by Eq. (5.5).

large momentum transfers: $|\mathbf{k}| \gg p_{F}$. In this limit, the particle-hole bubble becomes 14

$$
\Pi_{\mathrm{ph}}\left(\omega_{k}, \mathbf{k}\right)=-\frac{p_{F}^{2}}{2 \pi} \frac{E_{\mathbf{k}}}{\omega_{q}^{2}+E_{\mathbf{k}}^{2}},
$$

where $E_{\mathbf{k}}=\mathbf{k}^{2} / 2 m$. At fixed number density of particles $n$, the area of the Fermi surface is inversely proportional to the number of flavors

$$
p_{F}^{2} / 4 \pi=n / N
$$

With this normalization, the product $N \Pi_{\mathrm{ph}}\left(\omega_{k}, \mathbf{k}\right)=$ $-2 n E_{\mathbf{k}} /\left(\omega_{k}^{2}+E_{\mathbf{k}}^{2}\right)$ is independent of $N$. A pole of $\tilde{U}$ in real frequencies corresponds to the collective (zero-sound) mode with dispersion $\omega_{k}=\sqrt{E_{\mathbf{k}}^{2}+E_{0} E_{\mathbf{k}}}$, which interpolates between sound-like excitations for $E_{\mathbf{k}} \ll E_{0} \equiv 2 n U$ and particle-like excitations for $E_{\mathbf{k}} \gg E_{0}$. Note that $E_{0} / E_{F} \sim N U m \gg 1$.

According to Eq. (1.3), mass renormalization is determined by a stand-alone $\epsilon_{\mathbf{p}}$ term in the self-energy. This term is obtained by expanding the single-particle dispersion in the argument of the Green's function in Eq. (5.4) as $\epsilon_{\mathbf{p}+\mathbf{k}}=\epsilon_{\mathbf{p}}+\epsilon_{\mathbf{p}} \mathbf{p}_{F} \cdot \mathbf{k} / p_{F}^{2}+\mathbf{p}_{F} \cdot \mathbf{k} / m+E_{\mathbf{k}}$ and differentiating the regular part of the self-energy with respect to the second term in $\epsilon_{\mathbf{p}+\mathbf{k}}$ at $\epsilon_{\mathbf{p}}=\omega=0$. This yields

$$
\frac{m}{m^{*}}=1-\int \frac{d^{2} k}{(2 \pi)^{2}} \int \frac{d \omega_{k}}{2 \pi} G\left(\mathbf{p}_{F}+\mathbf{k}, \omega_{k}\right) \tilde{U}\left(\omega_{k}, \mathbf{k}\right) \frac{\mathbf{p}_{F} \cdot \mathbf{k}}{p_{F}^{2}} .
$$

Since, according to our assumption, large $|\mathbf{k}|$ control the integral in Eq. (5.8), the $E_{\mathbf{k}}$ term in the denominator of Green's function is larger than the $\mathbf{p}_{F} \cdot \mathbf{k}$ term. Expanding the Green's function to first order in $\mathbf{p}_{F} \cdot \mathbf{k}$, integrating over the angle, and switching from integration over $|\mathbf{k}|$ to 

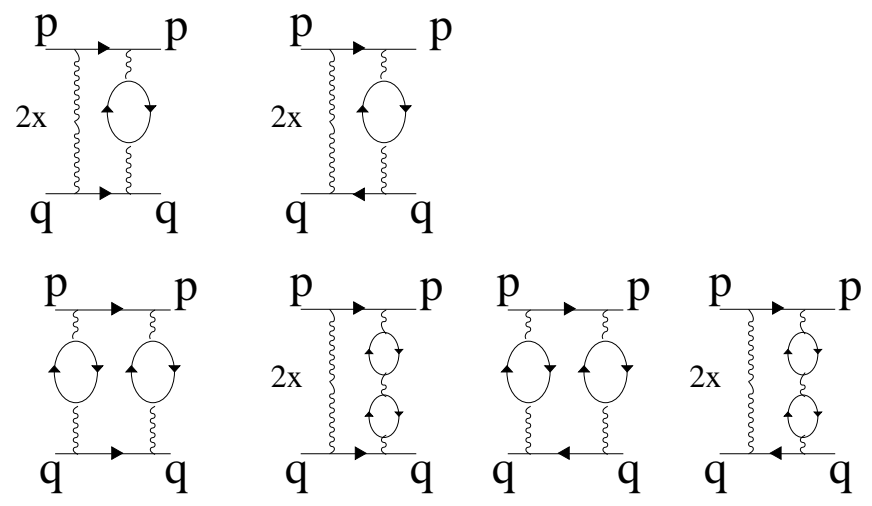

FIG. 4: Diagrams for the Fermi-liquid vertex $\Gamma_{\alpha \beta, \gamma \delta}^{\omega}(p, q)$ in the large- $N$ limit and to fourth order in the momentumindependent interaction $U=$ const. We select only those diagrams which contribute to mass renormalization in 2D. Note that particle-particle and particle-hole diagrams differ by the directions of arrows on bottom fermionic lines.

integration over $E_{\mathbf{k}}$, we obtain

$$
\begin{aligned}
\frac{m}{m^{*}}= & 1-\frac{U m}{2 \pi^{2}} \int_{0}^{\infty} d E_{\mathbf{k}} \int_{-\infty}^{\infty} d \omega_{k} \frac{\omega_{k}^{2}+E_{\mathbf{k}}^{2}}{\omega_{k}^{2}+E_{\mathbf{k}}^{2}+E_{0} E_{\mathbf{k}}} \\
& \times \frac{E_{\mathbf{k}}}{\left(i \omega_{k}-E_{\mathbf{k}}\right)^{3}}
\end{aligned}
$$

Now it is obvious that typical $\left|\omega_{k}\right| \sim E_{\mathbf{k}} \sim E_{0} \gg E_{F}$, which justifies our original assumption. Performing remaining integrations, we finally obtain

$$
\frac{m^{*}}{m}=1+\frac{m U}{8 \pi} .
$$

As it is also the case for the Coulomb interaction, $\stackrel{14,15}{t h e}$ effective mass in the $N U m \gg 1$ limit does not depend on $N$. Note that Eq. (5.10) is valid only for a repulsive and sufficiently weak interaction $(U m \ll 1)$, so that mass renormalization is still a small albeit non-perturbative effect. Notice also that the normalization condition (5.7) was not essential: if it is not imposed, the high-energy scale $E_{0}$ is replaced by $\tilde{E}_{0}=(N U m / \pi) E_{F} \gg E_{F}$. However, since the high-energy scale drops out from the formula (5.9) for the effective mass, changing $E_{0}$ by $\tilde{E}_{0}$ does not affect the result for $m^{*}$.

Differentiating the regular part of the self-energy with respect to $i \omega$ and performing integrations in a way similar to the effective mass case, we obtain the $Z$ factor in the $N U m \gg 1$ limit

$$
Z=1-\frac{m U}{4 \pi} .
$$

\section{Fermi-liquid formalism in the large- $N$ limit}

As we explained in Sec. VA to obtain the effective mass in the FL formalism, one needs to collect all $d i$ rect diagrams for $\Gamma^{\omega}$ to next-to-leading order in $1 / N$. This arduous task is simplified dramatically in the case of a momentum-independent interaction in 2D, where diagrams with particle-hole bubbles at $\mathbf{p}-\mathbf{q}$ do not contribute to mass renormalization. To second order in $U$, the leading order in $N$ is $N^{1}=N$ and next-to-leading order is $N^{0}=1$. There is only one direct, $\mathcal{O}\left(N^{0}\right)$ diagram that does not contain $\Pi_{\mathrm{ph}}(\omega=0, \mathbf{p}-\mathbf{q})$-diagram $c$ in Fig. 1] To third order in $U$, there are only two inequivalent diagrams of order $\mathcal{O}\left(N^{1}\right)$, shown in Fig. 4. One of them renormalizes the second-order particle-particle diagram $c$, while the other renormalizes the particle-hole diagram $e$ in Fig. 1] Note that the particle-hole bubbles in both diagrams are integrated over internal four-momenta and, hence, do contribute the angular dependence of $\Gamma^{\omega}$. To fourth order in $U$, there are four inequivalent diagrams, also shown in Fig. 4 etc. It is easy to see that the overall combinatorial coefficients for particle-particle and particle-hole diagrams of order $U^{n}$ are both equal to $n-1$. Collecting all orders, we obtain for the angulardependent part of $\Gamma^{\omega}$

$$
\begin{aligned}
\Gamma_{\alpha \beta, \gamma \delta}^{\omega}\left(p_{F}, q_{F}\right) & =-U^{2} \delta_{\alpha \beta} \delta_{\gamma \delta} \int \frac{d^{3} l}{(2 \pi)^{3}}\left[G_{p+l} G_{q+l}+G_{p+l} G_{q-l}\right] \sum_{n=0}^{\infty}(n+1)\left[N U \Pi_{\mathrm{ph}}(l)\right]^{n} \\
& =-U^{2} \delta_{\alpha \beta} \delta_{\gamma \delta} \int \frac{d^{3} l}{(2 \pi)^{3}}\left[G_{k+l} G_{q+l}+G_{k+l} G_{q-l}\right] \frac{1}{\left[1-N U \Pi_{\mathrm{ph}}(l)\right]^{2}},
\end{aligned}
$$

where we added the $\mathcal{O}\left(U^{2}\right)$ particle-hole diagram, which does not contribute to mass renormalization, to the right-hand side of Eq. (5.12). The result for $\Gamma_{\alpha \beta, \gamma \delta}^{\omega}$ is greatly simplified in the limit of $N U m \gg 1$. As in the previous section, we replace $\Pi_{\mathrm{ph}}(l)$ by its largemomentum asymptotic form (5.6) and expand the prod- ucts of Green's functions as

$$
\begin{aligned}
G_{p+l} G_{q+l} & =\frac{\left(v_{F} l\right)^{2}}{\left(i \omega_{l}-E_{1}\right)^{4}} \cos \theta_{p l} \cos \theta_{q l}+\ldots \\
G_{p+l} G_{q-l} & =-\frac{\left(v_{F} l\right)^{2}}{\left(\omega_{l}^{2}+E_{1}^{2}\right)^{2}} \cos \theta_{p l} \cos \theta_{q l}+\ldots
\end{aligned}
$$


where ... stand for angular-independent and higher order terms, $\theta_{k_{1} k_{2}} \equiv L\left(\mathbf{k}_{1}, \mathbf{k}_{2}\right)$, and, as before, $E_{\mathbf{l}}=l^{2} / 2 m$.
After trivial angular integration and some simplifications, we obtain

$$
\begin{aligned}
\Gamma_{\alpha \beta, \gamma \delta}^{\omega}\left(p_{F}, q_{F}\right)= & -\delta_{\alpha \beta} \delta_{\gamma \delta} \frac{U^{2} k_{F}^{2}}{\pi^{2}} \cos \theta_{p q} \int_{0}^{\infty} d E_{\mathbf{l}} \int_{-\infty}^{\infty} d \omega_{l} \frac{i \omega_{l} E_{\mathbf{l}}^{2}}{\left(i \omega_{l}-E_{\mathbf{l}}\right)^{2}} \frac{1}{\left(\omega_{l}^{2}+E_{\mathbf{l}}^{2}+E_{0} E_{\mathbf{l}}\right)^{2}} \\
& =\delta_{\alpha \beta} \delta_{\gamma \delta} \cos \theta_{p q} \frac{U}{2 N}
\end{aligned}
$$

Substituting Eq. (5.14) into the formula for the effective mass (5.1), we reproduce the result of the perturbation theory, Eq. (5.10).

To reproduce the perturbative result for $Z$, one needs to evaluate $\Gamma_{\alpha \beta, \gamma \delta}^{\omega}\left(p_{F}, q\right)$ for $q$ away from the FS. We did not attempt to do this.

\section{CONCLUSIONS}

In this paper, we analyzed an interplay between highand low-energy contributions to two fundamental Fermiliquid parameters- the quasiparticle $Z$-factor and effective mass $m^{*}$-obtained in two different ways: via a general FL formalism and via a diagrammatic perturbation theory. In both cases, $Z$ and $m^{*}$ are extracted from the fermionic self-energy. In the FL formalism, the selfenergy $\Sigma_{\mathrm{FL}}$ is obtained from the Pitaevskii identities for the derivatives of the Green's functions (following from the partcile number conservation and Galilean invariance) and expressed via an antisymmetrized FL vertex $\Gamma^{\omega}$. In the perturbation theory, the self-energy $\Sigma_{\text {pert }}$ is obtained in series of non-antisymmerized interaction $U(\mid \mathbf{k}) \mid$. To any order in $U(|\mathbf{k}|)$, the two self-energies are not identical when expressed in terms of fermionic Green's functions, but certainly yield the same expressions for $m^{*} / m$ and $Z$. We found, however, that identical results for $m^{*}$ in the two approaches are determined by different regions of energies. Whereas the FL-theory $m^{*}$ comes from low energies, i.e., from the vicinity of the Fermi surface, the perturbative $m^{*}$ includes, in general, contributions from both low- and high energies. Only the sum of the two contributions coincides with the FL result for $m^{*}$. We found that the equivalence of $m^{*} / m$ in the two approaches is based on a particular identity for the products of fermionic Green's functions, Eq. (4.6) which relates the low- and high-energy contributions to the effective mass. On the other hand, renormalization of $Z$ comes only from high-energy fermions in both approaches. We obtained the expression for $Z$ in a 2D Fermi liquid with short-range interaction.

We also analyzed the difference between the FLand perturbative approaches for a system of interacting fermions with $S U(N)$ symmetry in the limit of $N \gg 1$. We showed that mass renormalization in the diagrammatic formalism comes from high energies, and that equivalent expressions for the effective mass are obtained in the two formalisms only if one collects next-to-leading terms in the $1 / N$ expansion for $\Gamma^{\omega}$. We obtained a closed expression for $\Gamma^{\omega}$ for the case of momentum-independent interaction in $2 \mathrm{D}$.

The lesson to be learned from this consideration is that one has to be careful with eliminating high-energy fermions from the problem. While it is tempting to reduce the problem to that of low-energy fermions with an effective interaction and consider only the low-energy contribution to $m^{*} / m$, this would give an incorrect result for $m^{*}$. The reason is that, in the process of integrating out high-energy fermions, the quasiparticle mass changes from its bare value, $m$, to a new one, $m_{B}$. The difference $m_{B} / m-1$ comes from high energies. Only the the combined effect of high-energy renormalization, which replaces $m$ by $m_{B}$, and low-energy renormalization, which involves onlt the Fermi-surface states, yields the agreement with the FL theory.

\section{ACKNOWLEDGEMENT}

We acknowledge helpful discussions with E. Fradkin and R. Shankar. This work was supported by NSFDMR-0906953 (A. V. Ch.) and NSF-DMR-0908029 (D. L. M.). The authors acknowledge the support from MPIPKS, Dresden, where a part of this work was done. A.V. Ch acknowledges the hospitality of the Aspen Center for Physics.
1 A. A. Abrikosov, L. P. Gorkov, and I. E. Dzyaloshinski, Methods of quantum field theory in statistical physics, (Dover Publications, New York, 1963); E. M. Lifshitz and L. P. Pitaevski, Statistical Physics, (Pergamon Press, 1980).

2 D. Pines and P. Nozieres, The theory of quantum liquids, 
(Addison-Wesley, Menlo Park, 1966).

3 Anderson P.W. Basic notions of Condensed Matter Physics, (Benjamin-Cummings, Melno Park, 1984).

${ }^{4}$ G. Baym and C. Pethick, Landau Fermi Liquid theory, (Wiley, New York, 1991).

5 L. P. Pitaevskii, Sov. Phys. JETP 10, 1267 (1960).

${ }^{6}$ A. A. Abrikosov and I. M. Khalatnikov, Sov. Phys. JETP 10, 132 (1958);

7 V.M. Galitskii, Sov. Phys. JETP 7, 104 (1958).

8 R. Shankar, Rev. Mod. Phys. 66, 129 (1994).

9 To the best of our knowledge, the second-order result for $Z$ [Eq. [2.12] ] was not derived in the literature within the FL theory, i.e., using Eq. (1.2a).
10 See, e.g., V. M. Galitski and S. Das Sarma, Phys. Rev. B 70, 035111 (2004) and references therein.

11 G. Burkard, D. Loss, and E.V. Sukhorukov, Phys. Rev. B 61 R16303 (2000).

12 J. R. Engelbrecht, M. Randeria, and L. Zhang, Phys. Rev. B 45, R10135 (1992).

13 G. Y. Chitov and D. Sénéchal, Phys. Rev. B 52, 13847 (1995).

14 S. V. Iordanskii and A. Kashuba, JETP Lett. 76, 563 (2002).

15 S. Gangadharaiah and D. L. Maslov, Phys. Rev. Lett. 95, 186801 (2005). 